EPJ Web of Conferences 59, 18007 (2013)

DOI: $10.1051 /$ epjconf/20135918007

(C) Owned by the authors, published by EDP Sciences, 2013

\title{
THz radiation from an ultrashort-laser-induced fast spark dense plasma
}

\author{
N. Yugami ${ }^{1, a}$, F. Suzuki ${ }^{1}$, H. Kashiwazaki ${ }^{1}$, T. Higashiguchi ${ }^{1}$, Y. Sentoku ${ }^{3}$, \\ A. Nishida ${ }^{2,4}$ and R. Kodama ${ }^{2,4}$ \\ 1 Utsunomiya University, Department of Advanced Interdisciplinary Sciences, Yoto 7-1-2, \\ Utsunomiya, Japan \\ 2 Japan Science and Technology Agency, CREST, 4-1-8 Honcho, Kanagawa, Japan \\ 3 University of Nevada, Department of Physics, Mail Stop 220, Reno, Nevada, USA \\ ${ }^{4}$ Osaka University, Graduate School of Engineering, 2-6 Yamada-oka, Suita, Osaka, Japan
}

\begin{abstract}
We demonstrate a frequency-tuning scheme that uses laser pulse duration to control the ultrafastdischarge current timescale. We also propose a simple physical model to explain the generation of terahertz radiation by laser propagation in an ultrafast-discharge.
\end{abstract}

\section{INTRODUCTION}

Laser-produced filamentation in an electrostatic field has been reported as the basis for radiation generation through four-wave mixing, but without supporting information about the microscopic origin of the nonlinearity $[1,2]$. Electron plasma wave excitation by a low intensity ultrashort laser pulse in the linear region has also been proposed as a mechanism for a radiation source [3, 4]. However, when the laser intensity is low, i.e., the normalized vector potential much lower than unity, an electron plasma wave is not excited [5]. Plasma-based radiation sources using a laser-wakefield (i.e., with high intensity laser pulses), on the other hand, have the advantage of being frequency tunable, and of producing ultrashort, high-power pulses. Emission of $\mathrm{THz}$ radiation from an ultrashort laser-produced plasma has been demonstrated [6,7]. The plasma current model in the two color laser fields has been proposed to explain clearly by Kim and co-worker [8]. In this model the THz radiation originates from the nonzero drift motion of the electrons in the two color laser fields. Note that $\mathrm{THz}$ radiation with 0.1-0.3 THz has been observed in a single color laser field [9]. In addition, the radiation frequency was almost constant from laser-induced filaments in air with DC bias [10]. On the other hand, the model to explain the generation of $\mathrm{THz}$ radiation in air includes the plasma frequency [11]. As a result, the radiation frequency should change with the plasma frequency by changing the electron density or laser intensity. Many experimental demonstrations show the power scaling and output energy, but with fixed peak frequency, i.e., no tunable $\mathrm{THz}$ emission. However it was frequently reported that the peak frequency remained almost constant although the spectrum broadened with increasing laser intensity or electron density. We focus on the physical mechanism to produce tunable, high power $\mathrm{THz}$ radiation in an ultrashort-laser-induced discharge in air. To demonstrate the ultrafast discharge we developed a home-made pulse power supply with a 100-A peak current, 100-ns pulse duration at the repetition rate of $1 \mathrm{kHz}$. As we show later, the peak frequency strongly depends on the ionization time, that is, the duration of the laser pulse. This scheme is very simple. Understanding the laser

\footnotetext{
ae-mail: yugami@cc.utsunomiya-u.ac.jp
}

This is an Open Access article distributed under the terms of the Creative Commons Attribution License 2.0, which permits unrestricted use, distribution, and reproduction in any medium, provided the original work is properly cited. 


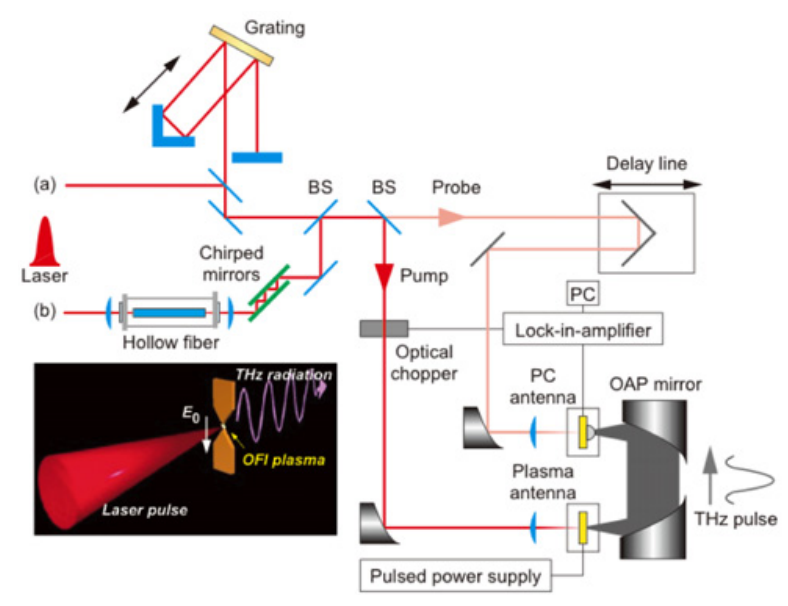

Figure 1. Experimental setup for $\mathrm{THz}$ generation. An ultrashort laser pulse with a maximum laser energy of $1 \mathrm{~mJ} /$ pulse is focused onto an electrode gap, connected to a pulsed power supply and produces $\mathrm{THz}$ radiation.

propagation in the gas provides knowledge of the fundamental basic electromagnetic dynamics in the ultrafast-discharge including ultrafast conductive current, electric-field-induced displacement current and polarization produced coherent radiation in THz to soft X-ray with half-cycle electric field.

An ultrafast-discharge is produced in air between a positively biased and a grounded electrode. When a plasma is produced by the ultrashort laser pulse at $t=0$, an electron sheath is created at the surface of the positive electrode and expands toward the grounded electrode. Its velocity scales as the thermal velocity of free electrons in the plasma. The electrons accelerate in the electron sheath. The electric field of the radiation strongly depends on the electron density profile of the sheath, i.e., the laser pulse duration and the ionization front shape. Therefore, the radiation frequency strongly depends on the duration of the ultrashort laser pulse.

\section{EXPERIMENT AND DISCUSSION}

\subsection{Experimental setup}

In our experiment a Ti:sapphire laser provided a pulse with a width of $100 \mathrm{fs}$ (FWHM) and a central wavelength of $800 \mathrm{~nm}$ that was split into a pump and a probe beam. Pulse durations up to $300 \mathrm{fs}$ could be generated by changing the angle of one of the grating pairs in the compressor of the laser system. Pulse durations from 30 to $70 \mathrm{fs}$ were generated by the nonlinear compression of the laser pulse by a hollow fiber and the chirp mirrors. The pump beam to produce the ultrafast discharge induced by the ultrashort laser pulse was focused at the gap where the electrostatic field strength was $28 \mathrm{kV} / \mathrm{cm}$ at a repetition rate of $1 \mathrm{kHz}$, and was synchronized with the laser pulse. The peak current in the cold test was $100 \mathrm{~A}$ at a repetition rate of $1 \mathrm{kHz}$. The focused intensity of the pump beam was maximized to be $I_{L}=1 \times 10^{15} \mathrm{~W} / \mathrm{cm}^{2}$. The laser pulse energy was adjusted to keep the intensity constant for all pulse durations. The temporal waveform and the frequency spectrum of the emitted radiation were detected by a widely used pump and probe system, with a photoconductive (PC) antenna as a detector (Fig. 1). The output energy was measured by a liquid He-cooled 4.2-K Si bolometer (Infrared Inc. HDL-5).

\subsection{Results and discussion}

To evaluate the central frequency of the THz radiation, the temporal history of the radiation was observed by a PC antenna, and the spectrum was obtained by Fourier transformation of the temporal waveform 

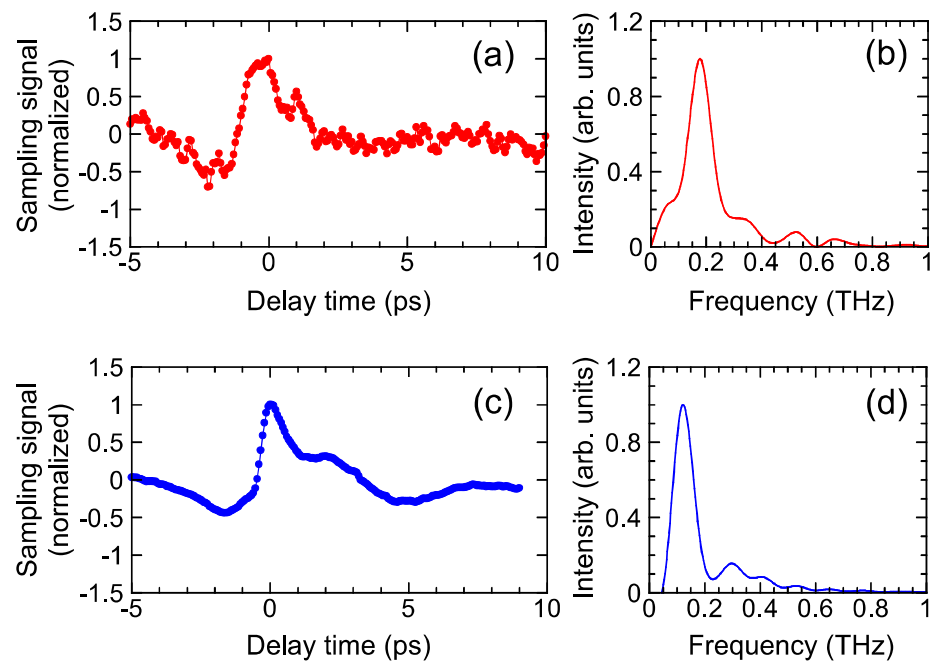

Figure 2. THz spectral observation from the ultrafast-discharge at different laser pulse durations. (a)-(d), Temporal histories of the THz radiation (a,c) and the corresponding spectra (b,d) at different laser pulse durations: $30 \mathrm{fs}(\mathrm{a}, \mathrm{b})$ and $100 \mathrm{fs}(\mathrm{c}, \mathrm{d})$.

of its electric field. The maximum detectable frequency was limited to $1 \mathrm{THz}$ due to the structure of the PC antenna detector. Figure 2(a)-2(d) shows the time history of the waveforms at laser pulse durations of 30 and $100 \mathrm{fs}$ and the corresponding $\mathrm{THz}$ spectra. The discharge $\mathrm{THz}$ source is an ideal point source. The peak frequency increases with decreasing laser pulse duration. The structure in the spectra originated from water vapor absorption in air. For the 30-fs laser pulse duration, the highest peak frequency component appeared around $0.25 \mathrm{THz}$.

To confirm that the plasma sheath current model in the ultrafast-discharge at the electrode gap describes the experimental results, we observed the laser pulse duration dependence on the peak frequency, which was evaluated from the spectra. In the case of the discharge, the time evolution of the plasma sheath is expected to be longer than the laser pulse duration, because the characteristic timescale for the plasma sheath is inversely proportional to the plasma frequency. According to the plasma sheath current model, the electron current in the ultrafast-discharge can be represented as $J=-\left[e n(t) D_{0} / \tau\right] \exp (-t / \tau)$, with $n(t)$ the temporal change of the electron density, $D_{0}$ is the static Debye length, and $t$ is the characteristic time and $\tau \propto \omega_{\mathrm{p}}^{-1}$. Here $\omega_{\mathrm{p}}$ is the plasma frequency and we assume that the one-dimensional (1D) sheath thickness is $D(t)=D_{0}[1-\exp (-t / \tau)]$. The electron current is dominated by the plasma density $n(t)$, because the term $\exp (-t / \tau)$ is almost constant as $t / \tau \ll 1$ under the experimental conditions. As a result, the plasma sheath current strongly depends on the temporal change of the electron density, $n(t)$, and thus the central frequency of the $\mathrm{THz}$ spectrum depends on the laser pulse duration. Furthermore, the output power decreases with shorter laser pulse durations, because the electric potential in the sheath near electrode decreases (electrons are born during the laser pulse).

Figure 3(a) shows the summary of the peak frequency as a function of the laser pulse duration. The radiation frequency depends on the temporal waveform of its electric field, i.e., the Fourier transformation of the waveform gives the frequency of the radiation. The electron density in the discharge plasma was almost constant for laser pulse durations from 30 to $300 \mathrm{fs}$ at a laser intensity of $5 \times 10^{14} \mathrm{~W} / \mathrm{cm}^{2}$ (Fig. 3(b)). The THz peak frequency scales with the reciprocal of the ionization time, i.e., the laser pulse duration, and this behavior, reproduced in the plasma sheath current model is shown in Fig. 4(b). As a matter of detail, the slight difference in the slopes at a boundary pulse duration of $100 \mathrm{fs}$ observed is attributed to the slight slope difference of $d n(t) / d t$ (Fig. 3(c)). 

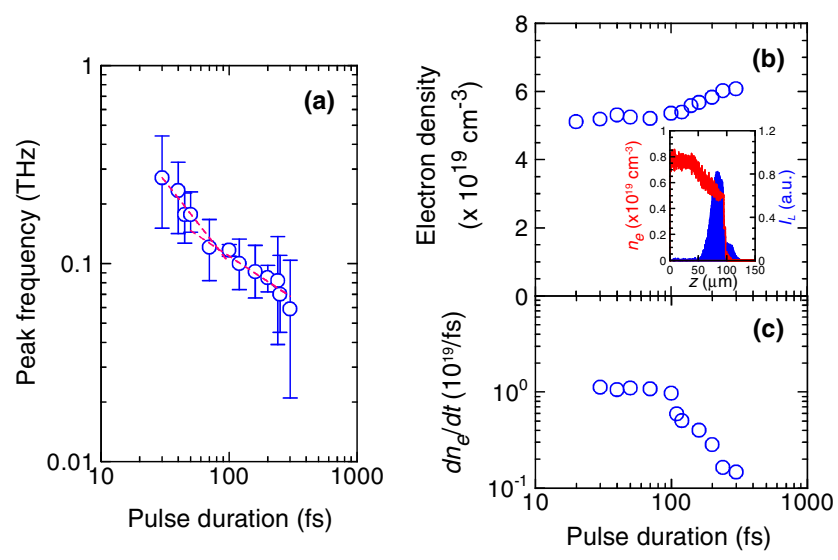

Figure 3. Laser pulse duration dependence. (a) The peak frequency increases with short laser pulse duration. (b) Electron density in ultrafast-discharge as a function of the laser pulse duration from a particle-in-cell (PIC) simulation. Inset: laser intensity waveform at the pulse duration of $100 \mathrm{fs}$ (blue) and the electron density (red) profiles at the discharge gap central. (c) The laser pulse duration dependence on the temporal change of the electron density from the PIC code. The slight difference of the slopes at a boundary of the pulse duration of $100 \mathrm{fs}$ is calculated.

\section{SUMMARY}

In summary, we have demonstrated a proof-of-principle peak-frequency-tunable $\mathrm{THz}$ radiation source based on laser-triggered plasma ultrafast-discharge. An unified physical picture for the peak frequencyversus laser pulse duration dependency in the discharge, based on the plasma sheath current model together with a plasma expansion driven by the OFI, was also presented.

\section{References}

[1] D. J. Cook and R. M. Hochstrasser, Opt. Lett. 25, 1210 (2000)

[2] X. Xie, J. Dai, and X.-C. Zhang, Phys. Rev. Lett. 96, 075005 (2006)

[3] A. Houard, Y. Liu, B. Prade, V. T. Tikhonchuk, and A. Mysyrowicz, Phys. Rev. Lett. 100, 255006 (2008)

[4] C. D. Amico, A. Houard, S. Akturk, Y. Liu, J. L. Bloas, M. Franco, B. Prade, A. Couairon, V. T. Tikhonchuk, and A. Mysyrowicz, New J. Phys. 10, 013015 (2008)

[5] E. Esarey, P. Sprangle, J. Krall, and A. Ting, IEEE Trans. Plasma Sci. 24, 252 (1996)

[6] H. Hamster, A. Sullivan, S. Gordon, W. White, and R. W. Falcone, Phys. Rev. Lett. 71, 2725 (1993)

[7] N. Yugami, T. Higashiguchi, H. Gao, S. Sakai, K. Takahashi, H. Ito, Y. Nishida, and T. Katsouleas, Phys. Rev. Lett. 89, 065003 (2002)

[8] K. Y. Kim, A. J. Taylor, J. Glownia, and G. Rodriguez, Nat. Photonics 2, 605 (2008)

[9] N. Yugami, K. Ninomiya, K. Kobayashi, and H. Noda, Jpn. J. Appl. Phys. 45, L1051 (2006)

[10] Y. Chen, T. J. Wang, C. Marceau, F. Théberge, M. Châteauneuf, J. Dubois, O. Kosareva, and S. L. Chin, Appl. Phys. Lett. 95, 101101 (2009)

[11] W.-M. Wang, Z.-M. Sheng, X.-G. Dong, H.-W. Du, Y.-T. Li, and J. Zhang, J. Appl. Phys. 107, $023113(2010)$ 